Akademie der Landwirtschaftswissenschaften der Deutschen Demokratischen Republik

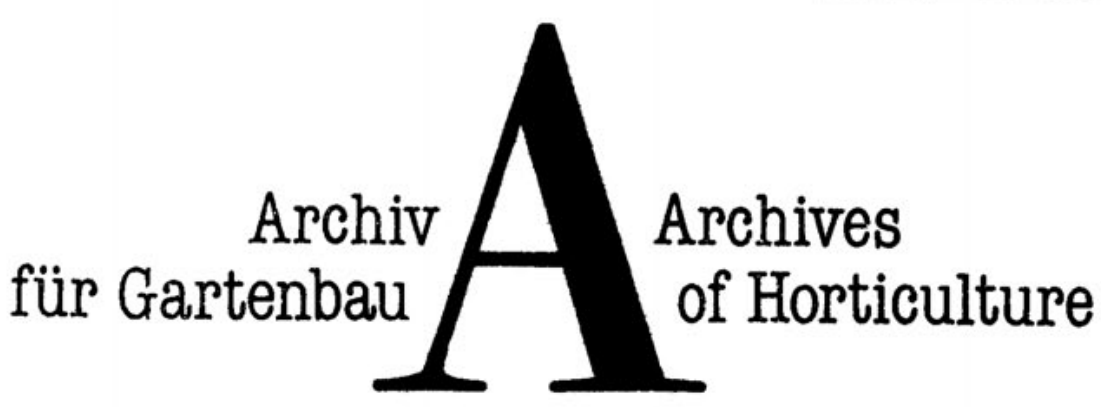

Volume $35 \cdot 1987 \cdot$ Number 7

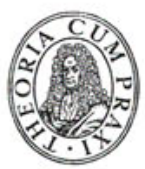

Akademie-Verlag · Berlin 
Zeitschrift „Archiv für Gartenbau“/"Archives of Horticulture"

Herauggeber: Akademie der Landwirtschaftswissenschaften

der Deutschen Demokratischen Republik

Krausenstraße 38/39, DDR - 1086 Berlin.

Verlag: Akademie-Verlag Berlin, Leipziger Straße 3-4, PF-Nr. 1233, DDR - 1086 Berlin.

Fernruf: 2236221 oder 2236229 , Telex-Nr.: 114420 ;

Bank: Staatsbank der DDR, Berlin, Kto.-Nr.: 6836-26-207 12.

Chefredakteur: Prof. Dr. sc. Wolfgang Frhrmann, Institut für Obstforschung Dresden-Pillnitz der AdL, Pillnitzer Platz 2, DDR - 8057 Dresden.

Redaktionskollegium: W. BIAsse, Marquardt; H. BocHOW, Berlin; H. FrÖHLICH, GroBbeeren; F. Gס̈HER, GroBbeeren; F. Katrmann, Berlin; H.-G. Katfmann, Berlin; H. KegleR, Aschersleben; A. Nisen, Gembloux; J. RUMPEL, Skierniewice; H. ROPPREcht, Berlin; G. STOLLE, Halle; G. VoGkL (gtellv Chefredakteur), Großbeeren; R. WhichoLD, Quedlinburg; S. J. WRRTHRTM, Wilhelminadorp; S. W. ZAGAJA, Skierniewice; H. ZmMerManN, Nossen.

Anschrift der Redaktion: Institut für Obstforschung Dresden-Pillnitz der AdL, „Archiv für Gartenbau“, Pillnitzer Platz 2, DDR - 8057 Dresden.

Veröffentlicht unter der Lizenznummer 1276 des Presseamtes beim Vorsitzenden des Ministerrates der Deutschen Demokratischen Republik.

Gesamtherstellung: VEB Druckerei „Gottfried Wilhelm Leibniz", DDR - 4450 Graffenhainichen

Erscheinungsweise : Die Zeitschrift „Archiv für Gartenbau“/"Archives of Horticulture” erscheint jăhrlich in einem Band mit 8 Heften. Das letzte Heft eines Bandes enthălt Inhalts-, Autoren- und Sachverzeichnis. Bezugspreis eines Bandes 200,- DM zuzüglich Versandspesen. Preis je Heft 25,- DM. Der gültige Jahresbezugspreis für die DDR ist der Postzeitungsliste zu entnehmen.

Bestellnummer dieses Heftes: 1039/35/7.

Urheberrecht: Die Rechte uber die in dieser Zeitschrift abgedruckten Arbeiten gehen ausschlieBlich an die Akademie der Landwirtschaftswissenschaften der Deutschen Demokratischen Republik über. Ein Nachdruck in anderen Zeitschriften oder eine tbersetzung in andere Sprachen bedarf der Genehmigung der Akademie, ausgenommen davon bleibt der Abdruck von Zusammenfassungen. Kein anderer Teil dieser Zeitschrift darf in irgendeiner Form - durch Photokopie, Mikrofilm oder ein anderes Verfahren - ohne schriftliche Genehmigung der Akademie reproduziert werden.

All rights reserved (including those of translation into foreign languages). No part of this issue, except the summaries may be reproduced in any form, by photoprint, microfilm or any other means, without written permission from the publishers.

(c) 1987 by Akademie-Verlag Berlin. Printed in the German Democratic Republic.

\section{AN (EDV) 48236}

00500

Bestellungen sind zu richten

- in der DDR an den Postzeitungsvertrieb unter Angabe der Kundennummer des Bestellenden oder an den AKADEMIE-VERLAG BERLIN, Leipziger Straße 3-4, PF-Nr 1233, DDR - 1086 Berlin;

- im sazialistischen Ausland an eine Buchhandlung für fremdsprachige Literatur oder an den zuständigen Postzeitungsvertrieb;

- in der BRD und Berlin (West) an eine Buchhandlung oder an die Auslieferungsstelle KUNST UND WISSEN, Erich Bieber OHG, WilhelmstraBe 4-6, D - 7000 Stuttgart 1;

- in den übrigen westeuropäischen Ländern an eine Buchhandlung oder an die Auslieferungsstelle KUNST UND WISSEN, Erich Bieber GmbH, General Wille-Str. 4, CH - 8002 Zürich;

- im Rbrigen Ausland an den Internationalen Buch- und Zeitschriftenhandel; den Buchexport, Volkseigener Außenhandelsbetrieb der Deutschen Demokratischen Republik, Postfach 160, DDR - 7010 Leipzig oder an den

AKADEMIE-VERLAG BERLIN, Lejpziger Straße 3-4, PF-Nr 1233, DDR - 1086 Berlin. 